\title{
SYNTHETIC MAPS OF THE BRIGHTNESS AND POLARIZATION OF THE F-CORONA
}

\author{
Y. FANG, P.L. LAMY, A. LLEBARIA \\ Laboratoire d'Astronomie Spatiale \\ Les Trois Lucs \\ 13012 Marseille, France
}

\begin{abstract}
The Wide-Field Light and Spectrometric Coronograph (LASCO) to be flown on SOHO in 1995 is designed to perform accurate photopolarimetric observations of the solar corona. For simulation purpose but also to have a two-dimensional model of the F-corona, we have realized synthetic maps of its brightness and polarization.
\end{abstract}

\section{Introduction}

The F-corona, which represents the inner extension of the zodiacal light, is intrinsically difficult to observe because of the proximity of the Sun and of the presence of the $K$ (electronic) corona. Carefull photopolarimetric measurements during total solar eclipses followed by an accurate separation of the $F$ and $K$ components are required to yield the radial variation of the brightness and polarization of the F-corona alone. Although the flattened aspect of both $\mathrm{K}$ and $\mathrm{F}$ coronae was perceived quite early, the non-sphericity of the K-corona only was analyzed and the ellipticity of the F-corona was traditionally neglected leading to classical spherical models (Van de Hulst, 1953; Saito, 1970). Recently, Koutchmy and Lamy (1985) re-analyzed various sets of observational data and produced a new, axially symmetric, non-spherical model in the form of two brightness profiles, equatorial and polar, from 1.1 to 30 solar radii $\left(R_{\theta}\right)$; in addition polarization values were derived up to $15 R_{\theta}$. Subsequent analysis by Baldayan (1988) have demonstrated the superiority of this model which is now generalized to produce full, two-dimensional maps of the brightness and polarization of the Fcorona.

\section{Procedure and observational data}

A key aspect in the analysis of Koutchmy and Lamy (1985) is the ellipticity of the corona as the shape of the observed isophotes are well fitted by ellipses. The present scheme to construct the two-dimensional maps is naturally based on this property and the equatorial and polar radial profiles allow to generate a family of elliptical iso-contours. This is done separately for the two polarized brightness, radial (or parallel) and tangential (or perpendicular). As the ellipticity decreases with the elongation, the model tends to sphericity in its most inner part,

A.C. Levasseur-Regourd and H. Hasegawa (eds.), Origin and Evolution of Interplanetary Dust, $195-198$. C 1991 Kluwer Academic Publishers, Printed in Japan. 
that is within $1.5 R_{\theta}$.

The two brightness profiles along the equatorial and polar directions of Koutchmy and Lamy (1985) were supplemented by zodiacal light data in order to insure a correct behaviour of the coronal brightness beyond $30 \mathrm{R}_{\theta}$ and to smoothly bridge to the zodiacal light. The procedure is exactly the same as that used by Lamy and Perrin (1986) except that additional data points were introduced inside $2.5 \mathrm{R}_{\theta}$. The data points were fitted by polynomials in log$\log$ form $\left(6^{\text {th }}\right.$ order) to give a functional representation of the brightness.

The polarization data of the F-corona and the zodiacal light have been fully analyzed and discussed by Lamy and Perrin (1986). We presently retain the polarization profiles appearing in their Fig. $8 \mathrm{c}$ (equatorial = plane of symmetry ; polar = meridian plane).

The construction of two-dimensional brightness images from the observed profiles is done in two steps. We first generate a field of elliptic isophotes with adequate spatial resolution. Then, for each pixel of the desired image, we interpolate the brightness between the adjacent isophotes.

\subsection{GENERATING THE FIELD OF ISOPHOTES}

The $\mathbf{k}^{\text {th }}$ isophote of the isophote field can be characterized by its semi-major axis $\mathbf{a}_{\mathbf{k}}$ (given by its intersection with the equatorial axis) and its semi-minor axis $b_{k}$ (given by its polar axis). Thus, the isophote field can be described by a set of pairs $\left(a_{k}, b_{k}\right)$ with $k=1, N$. For a given $a_{k}$, the corresponding $b_{k}$ is read from the brightness profiles. Rather than solving the transcendal equation $B_{\text {pol }}\left(b_{k}\right)=B_{\text {equ }}\left(a_{k}\right)$ for $b_{k}$, we interpolate $b_{k}$ from a table of values $\mathrm{B}_{\text {pol }}\left(\mathrm{r}_{\mathrm{i}}\right)$ taking advantage of the monotonic variation of $\mathrm{B}_{\mathrm{pol}}(\mathrm{r})$. The total number $\mathrm{N}$ of isophotes is taken as four times the desired resolution (e.g., $N=1024$ if a resolution of 256 pixels is selected). This is driven by the generation of the isophotes which do not intersect the equatorial and/or the polar axis and the requirement of obtaining a smooth variation of the coronal brightness.

\subsection{GENERATING THE BRIGHTNESS MAP}

Let $P\left(X_{i}, Y_{j}\right)$ be the center of the pixel $(i, j)$ where $X_{i}$ and $Y_{j}$ are the coordinates on the equatorial and polar axis. Two adjacent isophotes $(k-1)$ and $k$, can be found such that $P$ lies in between (Fig. 1). They satisfy:

$$
\mathrm{X}_{\mathrm{i}}{ }^{2 / \mathrm{a}_{\mathrm{k}}}{ }^{2}+\mathrm{Y}_{\mathrm{j}}{ }^{2} / \mathrm{b}_{\mathrm{k}}{ }^{2} \leqslant 1 \quad \mathrm{X}_{\mathrm{i}}{ }^{2 / \mathrm{a}^{2}{ }_{\mathrm{k}-1}}+\mathrm{Y}^{2}{ }_{\mathrm{j}} / \mathrm{b}^{2}{ }_{\mathrm{k}-1}>1
$$

The brightness $B\left(X_{i}, Y_{j}\right)$ at pixel $(i, j)$ is interpolated from these two isophotes according to the following cases.

i) Case A: For points such as $P_{A}$ (Fig. 1) satisfying $1-Y^{2} / b^{2}{ }_{k-1} \geqslant 0$ the interpolation is performed in the equatorial direction (this is the most frequent situation). We can find a point $P_{k-1}\left(X_{k-1}, Y_{j}\right)$ on the curve $(k-1)$, and another one $P_{k}\left(X_{k}, Y_{j}\right)$ on the curve $k$, such that

$$
X_{k-1}=a_{k-1} \sqrt{\left(1-Y^{2}{ }_{j} / b^{2}{ }_{k-1}\right)}, \quad X_{k}=a_{k} \sqrt{\left(1-Y_{j}^{2} / b^{2}{ }_{k}\right)}
$$


then, $B\left(X_{i}, Y_{j}\right)=B_{\text {equ }}(k-1)+\frac{X_{i}-X_{k-1}}{X_{k}-X_{k-1}}\left(B_{\text {equ }}(k)-B_{\text {equ }}(k-1)\right)$

ii) Case B: For points such as $P_{B}$ (Fig. 1) satisfying $1-Y_{j}^{2} / b^{2}{ }_{k-1}<0$ the point $\mathrm{P}_{\mathrm{k}-1}\left(\mathrm{X}_{\mathrm{k}-1}, \mathrm{Y}_{\mathrm{j}}\right)$ does not exist and the interpolation must be done in the polar direction. We can find a point $\mathrm{P}_{\mathrm{k}-1}\left(\mathrm{X}_{\mathrm{i}}, \mathrm{Y}_{\mathrm{k}-1}\right)$ on the curve $(\mathrm{k}-1)$, and another one $\mathrm{P}_{\mathrm{k}}\left(\mathrm{X}_{\mathrm{i}}, \mathrm{Y}_{\mathrm{k}}\right)$ on the curve $\mathrm{k}$, such that

$$
\begin{aligned}
& Y_{k-1}=b_{k-1} \sqrt{\left(1-X^{2}{ }_{j} / a^{2}{ }_{k-1}\right)}, \quad Y_{k}=b_{k} \sqrt{\left(1-X_{j}{ }_{j} / a^{2}{ }_{k}\right)} \\
& \text { then, } B(X i, Y j)=B_{e q u}(k-1)+\frac{Y_{i}-Y_{k-1}}{Y_{k}-Y_{k-1}}\left(B_{e q u}(k)-B_{e q u}(k-1)\right)
\end{aligned}
$$

Fig. 1. Determination of the brightness at a pixel

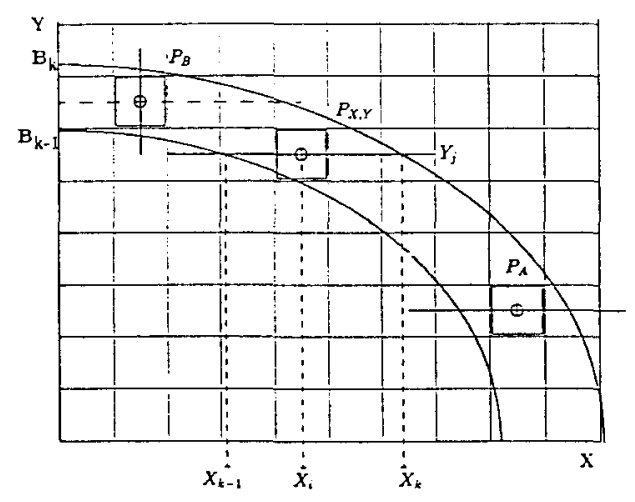

\section{Results}

The symmetry of the model allows to limit the calculations to one fourth of the corona. We present an example for a field-of-view of $30 \mathrm{R}_{\theta}$ with a resolution of $256 \times 256$ pixels. Fig. 2 gives the isophotes of the total brightness of the F-corona, the iso-contours of its polarization and the radial profiles of these two quantities along the equatorial and polar directions. One sees that the usual assumption of zero polarization made for the purpose of separating the $K$ and $F$ components of the corona becomes less and less valid as the elongation increases.

We are presently generating a model of the K-corona using however a different approach as we start from the spatial density of electrons and calculate the Thompson scattering. This model incorporates an homogeneous part as well as structures (Bohlin and Garrison, 1974). We shall then study the contrast of various Fraunhofer lines for the purpose of separating the $\mathrm{K}$ and $\mathrm{F}$-coronae. We shall also revisit the other method of separation based on polarization, relaxing the assumption of $P_{F}=O$ which is not valid in the outer corona. 

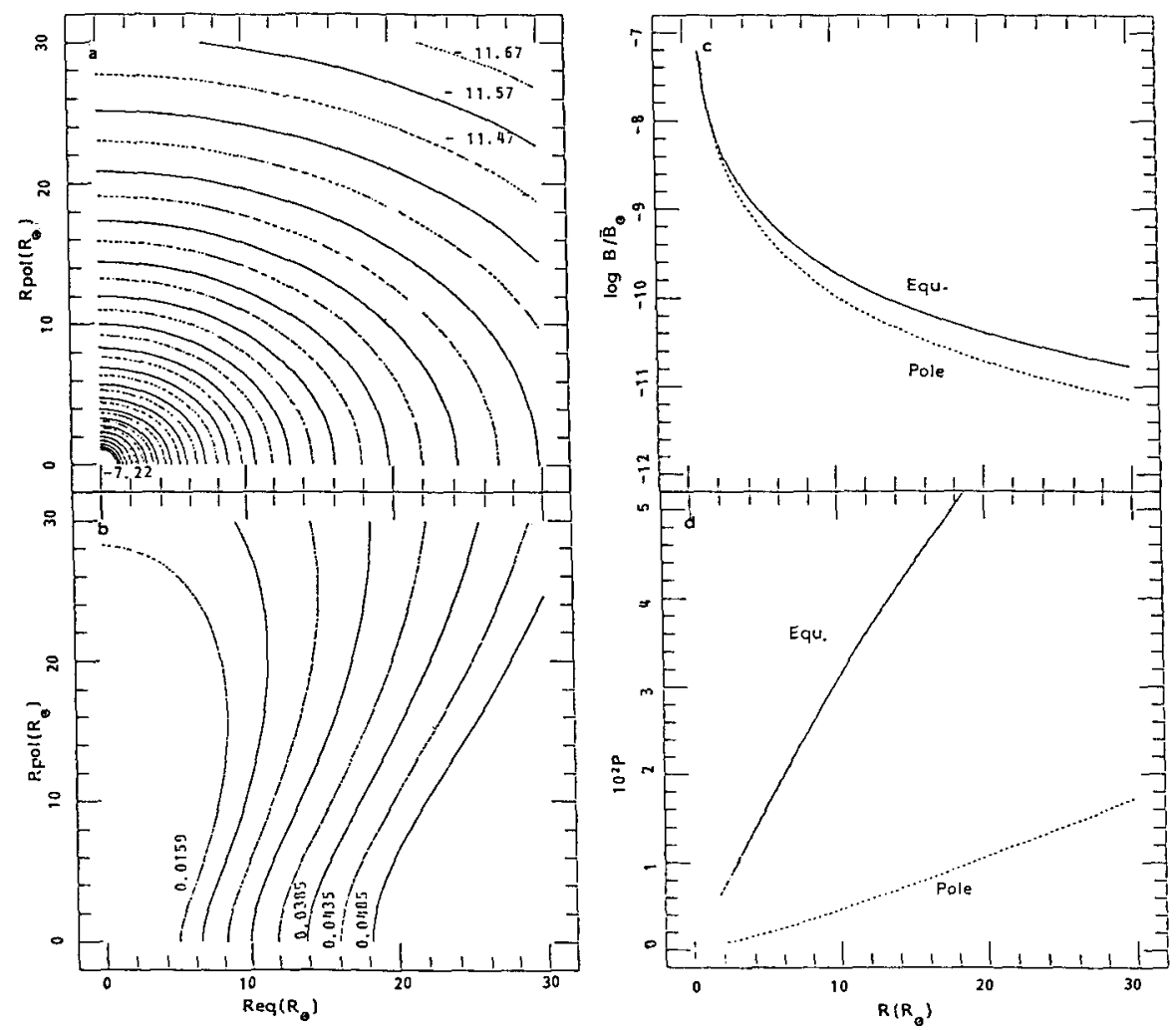

Fig. 2. The F-corona model out to $30 \mathrm{R}_{\odot}$ : a) isophotes of the total brightness (some values of $\log \mathbf{B}$ are indicated); b) isocontours of the polarization; the brightness profiles (c) and the polarization profiles (d) along the equatorial and polar directions.

\section{References}

Badalyan, O.G. (1988) The Physics of Solar Activity, Ed. Nauka, Moscow.

Bohlin, J.D. and Garrison, L.M. (1974) 'Numerical Calculation of Thompson Scattering from inhomogeneous models of the Corona', Solar Physics 38, 165.

Koutchmy, S. and Lamy, P.L. (1985) 'The F-corona and the Circum-Solar Dust: Evidences and Properties' in Properties and Interaction of Interplanetary Dust, eds. R. Giese, P. Lamy, pp. 63-73.

Lamy, P. and Perrin, J.-M. (1986) 'Volume Scattering Function and Space Distribution of the Interplanetary Dust Cloud', Astron. Astrophys, 163, 269.

Saito, K. (1970) 'A Non-spherical axisymmetric model of the solar K Corona of the Minimum Type', Ann. Tokyo Astron. Obs. Vol. XII, 2, pp. 53-120.

Van de Hulst, H.C. (1953) 'The Chromosphere and the Corona', in G.P. Kuiper (Ed.), The Sun, Univ. Chicago Press, Chicago, pp. 207-283. 